\title{
Benchmarking quantum control methods on a 12-qubit system
}

\author{
C. Negrevergne, ${ }^{1}$ T.S. Mahesh, ${ }^{2}$ C. A. Ryan, ${ }^{1}$ M. Ditty,${ }^{1}$ F. Cyr-Racine,${ }^{1}$ \\ W. Power ${ }^{1}$ N. Boulant, ${ }^{2}$ T. Havel, ${ }^{2}$ D.G. Cory ${ }^{2}$ and R. Laflamme ${ }^{1,3}$ \\ ${ }^{1}$ Institute for Quantum Computing, \\ University of Waterloo, Waterloo, ON, N2L 3G1, Canada. \\ ${ }^{2}$ Department of Nuclear Engineering, MIT, \\ Cambridge, Massachusetts 02139, USA \\ ${ }^{3}$ Perimeter Institute for Theoretical Physics, Waterloo, ON, N2J 2W9, Canada
}

(Dated: February 1, 2008)

\begin{abstract}
In this letter, we present an experimental benchmark of operational control methods in quantum information processors extended up to 12 qubits. We implement universal control of this large Hilbert space using two complementary approaches and discuss their accuracy and scalability. Despite decoherence, we were able to reach a 12-coherence state (or 12-qubits pseudo-pure cat state), and decode it into an 11 qubit plus one qutrit labeled observable pseudo-pure state using liquid state nuclear magnetic resonance quantum information processors.

PACS numbers: 03.67.Lx
\end{abstract}




\section{INTRODUCTION}

Quantum mechanics promises information processors that are more efficient than any known classical devices. However, to bring this potential to reality we must learn how to control large quantum systems in a scalable fashion. Scalability has at least two components: the complexity of the methods used to obtain coherent control must grow only polynomially with the number of qubits involved, and the errors occurring during the implementation of the control sequence must be small enough to be correctable. These errors can be split in two classes: first the operational errors due to imperfections in the control procedure and second, intrinsic errors due to decoherence and relaxation processes. Benchmarking small Quantum Information Processor (QIP) prototypes 1, 2, 3, 4] is therefore crucial to characterizing the errors in a physical system and developing general quantum control methods. In a physical system well suited for implementing a QIP, once we have reached an optimal operational control, one we will need to take care of intrinsic errors using quantum error correction procedures [5].

Because they have the ability to run non trivial quantum algorithms, liquid state Nuclear Magnetic Resonance (NMR) based QIPs 6, 1] can be used as benchmark systems [1, 8, 9]. In the present work we are interested in optimizing operational control strategies in terms of accuracy and amount of required classical resources. To do so we have chosen to extend the benchmarking algorithm previously used on a 7-qubit liquid state NMR register [1], to 11-qubit-plus-one-qutrit (see Fig.1). This algorithm consists of preparing mixtures of generalized GHZ states of the form: $\rho_{G H Z}=\mathbf{I}^{\otimes n}+\mathbf{X}^{\otimes n}$ ( $\mathbf{I}$ is the identity matrix, $\mathbf{X}$ is the $\boldsymbol{\sigma}_{\boldsymbol{x}}$ Pauli matrix and $n$ is the number of qubits involved in the GHZ states). This state preparation is very similar to the generation of stabilizer operators [10], which are building-blocks for quantum error correction codes. Furthermore, this algorithm takes the state of the quantum system to the most fragile reaches of the Hilbert space we are operating in, and therefore clearly demonstrates coherent control. Previous work has demonstrated a 12-spin pseudo-cat state [1] and multiple quantum coherences of much

higher order [12]. However, these exploited symmetries in the systems which limited them to a much smaller symmetric subspace of the full Hilbert space. In the present Letter we benchmark universal control methods that allow us to access the full Hilbert space of our system. 


\section{COHERENT UNIVERSAL CONTROL SCHEMES}

In liquid state NMR QIP, universal control is achieved through the application of a coordinated sequence of radio-frequency $(\mathrm{RF})$ pulses and periods of free evolution. The resulting one and two-qubit gates allow us, in principle, to implement any unitary transformation [13]. The challenge is to efficiently design such pulse sequences to be as short as possible and robust against experimental imperfections in order to minimize systematic error and decoherence [14, 15]. In a three-qubit experiment [16] it is possible to write the pulse sequences by hand and compensate for experimental errors with a few optimization parameters. Moving to larger registers [1, 8] requires more complex control schemes that necessitate systematic numerical optimization in the design of the pulse sequence. Going to 12-qubits represents a substantial step forward in the number of quantum degrees of freedom that are controlled.

We will approach coherent control over this large Hilbert space system from two complementary points of view. First, to demonstrate that control methods of high precision are available and experimentally realizable, we build a detailed model of the experimental QIP and for each desired unitary operation, search for an optimal control sequence based on strongly modulating pulses [17]. Applied over the entire Hilbert space, this approach is not scalable. The amount of classical resources required to search for control sequences grows as the size of the Hilbert space - i.e. exponentially with the number of qubits. However, this approach returns control sequences of high fidelity and with small, known errors, provided our system model is accurate. Because of the exponential computing cost of determining a suitable pulse, when dealing with a large Hilbert space we have to lower the dimension of the space over which we search. This can be achieved by searching for pulses only on a sub-system of the spins of the register (in the present case the carbon nuclear spins) and check that it leads to sufficiently high fidelity control by simulating the effect of rest of the spins as described in [18].

A second approach to control such a large Hilbert space is to make a series of wellconstructed simplifications to the model, in order to permit control sequences to be developed with a complexity that grows only polynomially with the number of qubits. We 
therefore based the design of our pulse sequences on the method developed in [1]. Indeed, by using only simple pulses (broad-band rectangular hard pulses and selective soft Gaussian shaped pulses) and performing a series of simulations on pairs of spins with significant couplings for each pulse, it is possible to efficiently determine first order deviations from the ideal pulse. For each of the pulses, these control errors can be represented as phase shifts and spin-spin coupling effects occurring before and after an ideal pulse. One can then modify the phase of each pulse to correct for the phase shifts. Assuming that long range couplings between the spins vanish, the timing between pulses can be efficiently numerically optimized in order to absorb the coupling effects into the refocusing scheme 19]. This design does not take higher-order coupling and off-resonant effects into account and leave some small couplings un-refocused to minimize the pulse sequence length. These approximations lead to errors in the control. A crucial point of this experimental work was to verify that these approximations hold for larger Hilbert spaces i.e. that we could find a suitable refocusing scheme that, once optimized, still provides reliable control.

\section{EXPERIMENTAL RESULTS AND DISCUSSIONS}

In liquid state NMR, the thermal equilibrium state is almost completely mixed. Therefore, instead of preparing $\rho_{G H Z}$ we actually prepare the following state:

$$
\rho_{c s} \simeq \frac{\mathbf{I}^{\otimes N}}{2^{N}}+\epsilon \mathbf{X}^{\otimes n} \mathbf{I}^{\otimes(N-n)}
$$

$N=14$ is the total number of spins- $1 / 2$ in the register, and $n$ is the number of qubits involved in the GHZ state. The factor $\epsilon \simeq 10^{-5}$ is related to the thermal polarization of the system. The second term of $\rho_{c s}$, called the deviation density matrix, contains the n-coherence term $|00 \ldots 0\rangle\langle 11 \ldots 1|+| 11 \ldots 1\rangle\langle 00 \ldots 0|$ corresponding to a $n$-qubit cat-state $|00 \ldots 0\rangle+|11 \ldots 1\rangle$, as well as lower coherence terms corresponding to the other n-qubit GHZ states. This state preparation (called the encoding of the pseudo-cat state) is done by propagating the polarization of the two equivalent protons through the chain of nuclei by a sequence of one and two-qubit quantum gates (see Fig. 1). In NMR only single coherence terms are observable 20]. Therefore, to see the signature of the GHZ state we need transform the

n-coherence term into a n-qubit labeled pseudo-pure state of the form X00....0 (where 
$\mathbf{0}=(\mathbf{I}+\boldsymbol{Z}) / 2)$. This step of the algorithm is called the decoding. To average away the other lower coherence order terms present in the $\mathbf{X}^{\otimes n}$ operator, we used two types of coherence filters: gradient and phase cycling techniques. Proof that we have actually created the pseudo-pure and accompanying pseudo-cat state by determining the final state through tomography would require an impractically large number of experiments $\left(\sim 4^{12}\right)$. Nevertheless, since the averaging procedure filters out signal coming from every term but the desired one (i.e. the highest coherence order term), a single observation of the "read out" nucleus in the resulting NMR spectrum (see Fig. 1) indicates that we have indeed reached the desired coherence.

We applied both methods to design two series of pulse sequences that implement the encoding-decoding procedure, with n going from 1 to 12, on a liquid state NMR QIP, based on uniformly ${ }^{13} C ;{ }^{15} N$ labeled l-histidine (See Fig. 2). Two different samples were used. The one used for designing strongly modulating pulses was made of $16.7 \mathrm{mg}$ of histidine, $15.9 \mathrm{mg}$ of deuterated phosphoric acid in $1 \mathrm{ml}$ of deuterated water. To design simplified pulse sequences we prepared a second sample by dissolving $35.3 \mathrm{mg}$ of histidine, $12.5 \mathrm{mg}$ of glycine- $2-{ }^{13} C ; ;^{15} N$ and $3.4 \mathrm{mg}$ of deuterated phosphoric acid in $1 \mathrm{ml}$ of deuterated water. The labeled glycine molecule has a simple spectrum which allowed us to perform accurate calibrations of the selective pulses on isolated NMR peaks in situ. The experiments based on the strongly modulating pulses and the simplified design were respectively performed on Avance-600 and Avance-700 Bruker spectrometers at MIT and IQC.

We based the design and the interpretation of the experiments on a model of the system and the apparatus [18] which includes the following attributes: (1) The Hamiltonian of the system in the static magnetic field of the spectrometer. The chemical shifts as well as the scalar-coupling strengths and relatives signs were experimentally determined by fits of reference spectra and small targeted multiple coherence experiments. (2) Knowledge of $T_{2}$ and $T_{2}^{*}$ 20] relaxation times of the system. (3) RF field inhomogeneities were mapped and used in the design the strongly modulating pulses [21].

This type of experiment comes with a predicted exponential decay of signal as we increase the number of correlated qubits. We also expect high decoherence rates [22, 23] and therefore a strong signal attenuation, as it is reasonable to assume that the relaxation rate for each spin included in the multiple quantum coherence add. To evaluate the quality of the control we could reach, the relevant quantity to measure is the amount of signal 
obtained experimentally with respect to the expected one assuming perfect control. Fig. 3 shows how much signal we were able to retain after decoding the highest coherence order cat state into a pseudo-pure state for each experiment. We could reach a 12-coherence state using strongly modulating pulses and a 10-coherence state with selective pulses. Indeed, the sequences obtained through the simplified design were slightly longer, leading to more

decoherence. Moreover, the transverse relaxation times were not the same in both sets of experiments. To distinguish between operational-errors and relaxation loss, both decay times $\left(T_{2}^{*}\right.$ and $\left.T_{2}\right)$ were used to estimate the signal loss due to transversal relaxation during the pulse sequences (See Fig.4). It showed that decoherence is the main source of signal loss and therefore indicates that we have good operational control.

\section{CONCLUSION}

In summary, we have reported an algorithmic benchmark performed on the largest quantum information processor to date. Despite the decoherence during the computation, we have been able to demonstrate universal control on up to 11 qubits and one qutrit. This work shows that liquid state NMR allows us to develop operational control methods that can be used to control a large number of quantum degrees of freedom. These methods provide a systematic and efficient way of programming liquid state NMR QIPs. However, the approaches and control techniques behind these methods could also be used to design control sequences in more scalable implementations where the intrinsic errors are smaller.

This work was supported by ARDA, ARO, LPS, NSERC and by the Cambridge-MIT Institute.

[1] E. Knill, R. Laflamme, R. Martinez, and C.-H. Tseng, Nature 404, 368 (2000).

[2] A. Furusawa, J. Sorensen, S. L. Braunstein, C. Fuchs, H. J. Kimble, and E. S. Polzik, Science 282, 706 (1998).

[3] M. Riebe, H. Haffner, C. F. Roos, W. Hansel, J. Benhelm, G. P. T. Lancaster, T. W. Korber, C. Becher, F. Schnidt-Kaler, D. F. V. James, et al., Nature 429, 734 (2004). 
[4] M. D. Barret, J. Chiaverini, T. Schaetz, J. Britton, W. M. Itano, J. D. Jost, E. Knill, C. Langer, D. Leibfried, R. Ozeri, et al., Nature 429, 737 (2004).

[5] D. P. DiVincenzo and P. W. Shor, Phys. Rev. Lett. 77, 3260 (1996).

[6] N. Gershenfeld and I. L. Chuang, Science 275, 350 (1997).

[7] D. G. Cory, A. F. Fahmy, and T. F. Havel, Proc. Natl. Acad. Sci. USA 94, 1634 (1997).

[8] L. M. K. Vandersypen, M. Steffen, G. Breyta, C. S. Yannoni, M. H. Sherwood, and I. L. Chuang, Nature 414, 883 (2001).

[9] E. Knill, R. Laflamme, R. Martinez, and C. Negrevergne, Phys. Rev. Lett. 86, 5811 (2001).

[10] D. Gottesman, Ph.D. thesis, California Institute of Technology, Pasadena, CA (1997), quant$\mathrm{ph} / 9705052$.

[11] J.-S. Lee and A. K. Khitrin, J. Chem. Phys. 122 (2005).

[12] W. S. Warren, D. P. Weitekamp, and A. Pines, J. Chem. Phys. 73, 2084 (1980).

[13] D. Barenco, C. H. Bennett, R. Cleve, D. P. DiVincenzo, N. Margolus, P. Shor, T. Sleator, J. Smolin, and H. Weinfurter, Phys. Rev. A 52, 3457 (1995).

[14] N. Khaneja, R. Brockett, and S. J. Glaser, Phys. Rev. A 63 (2001).

[15] J. A. Jones, Phys. Rev. A 67 (2003).

[16] D. Cory, M. D. Price, W. Maas, E. Knill, R. Laflamme, W. Zurek, T. F. Havel, and S. S. Somaroo, Phys. Rev. Lett. 81, 2152 (1998).

[17] E. M. Fortunato, M. A. Pravia, N. Boulant, G. Teklemariam, T. F. Havel, and D. G. Cory, J. Chem. Phys. 116, 7599 (2002).

[18] Y. S. Weinstein, T. F. Havel, J. Emerson, N. Boulant, M. Saraceno, S. Lloyd, and D. G. Cory, J. Chem. Phys. 121, 6117 (2004).

[19] M. D. Bowdrey, J. Jones, R. Laflamme, and M. Knill (2005), quant-ph/0506006.

[20] R. R. Ernst, G. Bodenhausen, and A. Wokaun, Principles of Nuclear Magnetic Resonance in One and Two Dimensions (Oxford University Press, Oxford, 1994).

[21] M. Pravia, N. Boulant, J. Emerson, A. Farid, E. Fortunato, T. Havel, R. Martinez, and D. Cory, J. Chem. Phys. 119, 9993 (2003).

[22] H. Krojansky and D. Suter, Phys. Rev. Lett. 93, 090501 (2004).

[23] J.-S. Lee and A. K. Khitrin (2005), quant-ph/0508052. 




FIG. 1: Sequence of gates for the 10-qubit pseudo-cat state preparation followed by its decoding into a 10-qubit pseudo-pure state. The initial preparation of the qutrit into a pseudo-pure state, as well as the refocusing gates, are not shown. Proper cycling of the Z-rotations and the phase of observation act as a coherence filter. The qubits names correspond to the histidine molecule nuclei (see Fig.2) a) After the qutrit pseudo-pure preparation, the state of the register is $\mathbf{0}^{H_{4 / 5}} \mathbf{I}^{H_{1}} \mathbf{I}^{C_{6}} \mathbf{I}^{N_{2}} \mathbf{I}^{H_{2}} \mathbf{I}^{C_{5}} \mathbf{I}^{C_{4}} \mathbf{Z}^{C_{3}} \mathbf{I}^{C_{2}} \mathbf{I}^{H_{3}} \mathbf{I}^{C_{1}}$. At the end of the encoding in b) it is 0XXXXXXXXXX and after filtering, the decoded state in c) is 0000X000000. 


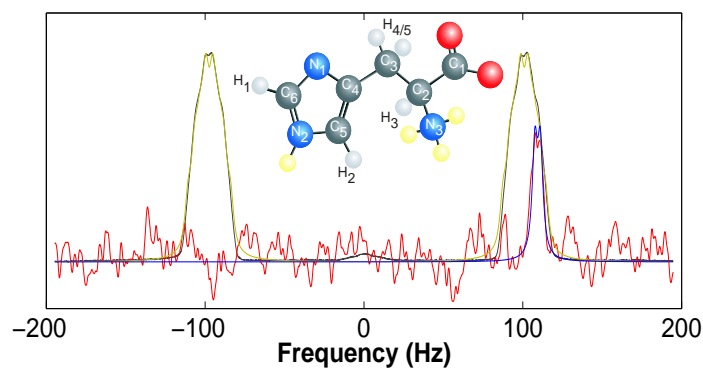

FIG. 2: This l-histidine molecule has 14 spin-1/2 nuclei: five ${ }^{1} H$, six ${ }^{13} C$ and three ${ }^{15} N$. See EPAPS Document No.1 for a more complete description of the molecule. The two protons $H_{4}$ and $H_{5}$, are chemically equivalent and indistinguishable. As such, they can be seen as an composite particle with a spin-1 and a spin-0 part. We considered only the spin-1 sub-space (qutrit) since the spin-0 does not interact with the other spin-1/2. This molecule is therefore a 12 -qubits plus one qutrit quantum register. However, the $N_{3}$ nuclear spin has a particularly weak coupling with the rest of the molecule; thus we did not use it. On this plot we have shown a reference spectrum of $H_{2}$ (gray plot) and of the pseudo-pure state obtained after decoding a 10-qubit cat-state onto $H_{2}$ (red line). They are arbitrarily scaled for clarity. The reference spectrum was obtained with 2 scans and the pseudo-pure, with 4000 scans, in order to improve the signal to noise ratio. We also show simulated spectra of the expected reference (yellow plot) and pseudo-pure state (blue line) for which the amplitude is matched to the experimental data to evaluate the signal loss. 


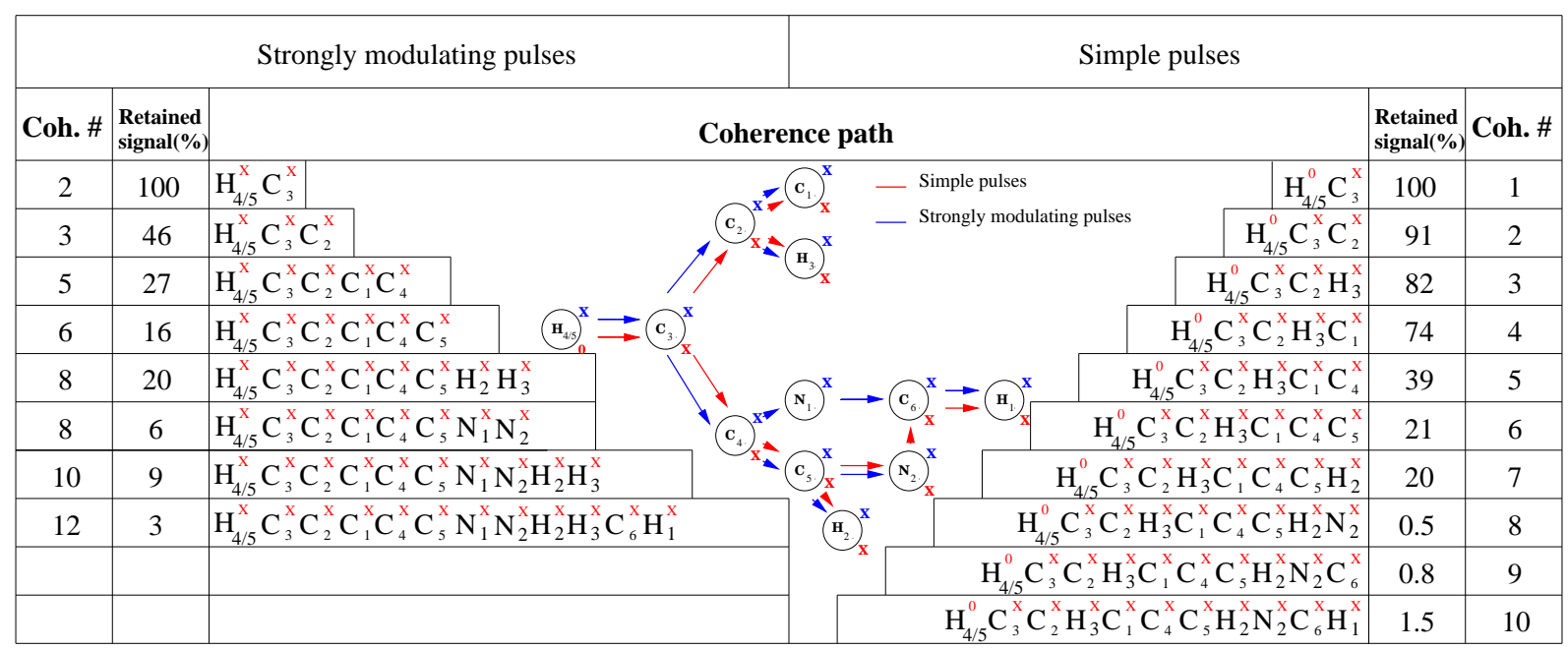

FIG. 3: Description of each of the multiple-coherence experiments. The picture shows how the polarization of the $H_{4 / 5}$ is propagated through the nuclei chain to create the cat state. In the series of experiments using simple pulses we first prepared the qutrit made of the two equivalent protons into a pseudo-pure state $\mathbf{0}=(\mathbf{I}+\boldsymbol{Z}) / 2$ and left it as such for the rest of the experiments. When using strongly modulating pulses, we included the qutrit into the multiple coherence state. The two first and two last columns show the coherence number we reached and how much signal we where able to retain after decoding the cat-state into a observable pseudo-pure state. Results are shows in $\%$ of the expected signal assuming perfect control, normalized to the first experiment. 


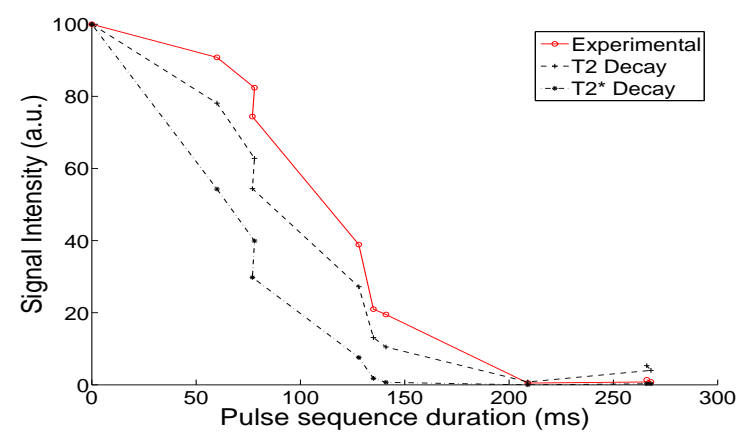

FIG. 4: Expected decay of the pseudo-pure state signal due to transversal relaxation for the series of experiments done with simple pulses. Each point correspond to a different coherence experiment. The length of the pulse sequence increases with the coherence order. Most experimental points are above the estimates given by $T_{2}^{*}$ and even $T_{2}$. Indeed, to predict the transversal relaxation during the pulse sequence, we only used a very simple model of decay for multiple coherences that gives an upper-bound of the signal loss. Nevertheless, the experimental curve and predicted ones show the same decay pattern. Thus it is reasonable to say that most of the signal loss comes from decoherence and therefore that we have a good operational control over the system. For the coherences 8, 9 and 10 (three last points), the experimental curve goes below the $T_{2}$ curve. It reflects a loss of accuracy in our operational control. Indeed, for these experiments, we are controlling the nitrogen nuclei through very small couplings. We are therefore using long two-qubits gates that are sensitive to any small inaccuracy in the values of the Hamiltonian parameters. 